\title{
In-volume structuring of silicon using picosecond laser pulses
}

\author{
H. Kämmer ${ }^{1} \cdot$ G. Matthäus ${ }^{1} \cdot$ S. Nolte ${ }^{1,2} \cdot$ M. Chanal ${ }^{3} \cdot$ O. Utéza $^{3} \cdot$ D. Grojo ${ }^{3}$
}

Received: 22 December 2017 / Accepted: 2 March 2018 / Published online: 13 March 2018

(c) The Author(s) 2018

\begin{abstract}
We have demonstrated for the first time the permanent local modification of the bulk of silicon by repeated illumination with infrared $(1.55 \mu \mathrm{m})$ picosecond pulses. Furthermore, we evaluated the characteristics of inscribing permanent modifications in the bulk material for different pulse durations from 0.8 to $10 \mathrm{ps}$ in terms of their reproducibility and controllability of their morphology. Our results are based on a simple experimental setup that demonstrates the possibility of using picosecond pulses for the local modification of bulk silicon as a potential alternative to more complex irradiation strategies required for femtosecond pulse processing.
\end{abstract}

\section{Introduction}

Today, in-volume structuring of different dielectric materials using ultrashort laser pulses is an established method for the generation of buried 3D optical elements like waveguides or Bragg gratings [1, 2]. In the case of silicon, strong nonlinear effects like multi-photon absorption in combination with plasma effects cause significant losses and a strong delocalization of the incident light before reaching the focus [3-5]. Consequently, for more than one decade in-volume processing of silicon with ultrashort laser pulses was only demonstrated close to the front [6] or rear [7] surface, thus strongly limiting the laser writing capabilities.

Recently, the processing of buried structures deep inside silicon by using ns-pulses and fs-pulses has been demonstrated [8-11]. Two publications show the generation of permanent structures deep inside silicon with the help of femtosecond laser pulses. The first approach is based on single pulses tightly focused at the center of silicon spheres for extreme solid-immersion focusing. Indeed this strategy reduces the strong nonlinear prefocal effects which prevent

H. Kämmer

helena.kaemmer@uni-jena.de

1 Institut für Angewandte Physik, Friedrich-SchillerUniversität Jena, Albert-Einstein-Straße 15, 07745 Jena, Germany

2 Fraunhofer Institut für Angewandte Optik und Feinmechanik IOF, Albert-Einstein-Straße 7, 07745 Jena, Germany

3 Aix Marseille University, CNRS, LP3, UMR7341, 13288 Marseille, France the delivery of intensities exceeding the modification threshold in conventional experiments [9]. The second approach demonstrates the writing of buried waveguides using femtosecond laser pulses at $250 \mathrm{kHz}$ repetition rate [11] assuming thermal effects for material modification. In practice, Pavlov et al. first induced a permanent modification close to the backside of a planar silicon sample and then moved from this seed modification the focus position towards the front surface along the optical axis for the longitudinal writing of guiding structures.

In this work, we report on permanent modifications achieved by multi-pulse irradiation inside planar Si samples with different pulse durations in the range from 0.8 to $10 \mathrm{ps}$ at repetition rates up to $200 \mathrm{kHz}$. With this work, we show that a simple writing setup for modifying silicon is sufficient. However, the controllability of laser-induced bulk modifications still needs to be improved to address the requirements for future technological applications.

\section{Experimental setup}

The experimental setup consists of a laser system for processing and an in situ lateral observation diagnostics (see Fig. 1). The experiments are carried out with an Er-doped fiber laser system (Raydiance, Inc.) operating at 1552 $\mathrm{nm}$ wavelength. This laser delivers maximum pulse energies between 80 and $30 \mu \mathrm{J}$ depending on the repetition rate $(30-200 \mathrm{kHz})$. In addition, the pulse duration of the laser source is adjustable from 0.8 to $10 \mathrm{ps}$ by detuning the built-in compressor stage. An overview of the range of the 


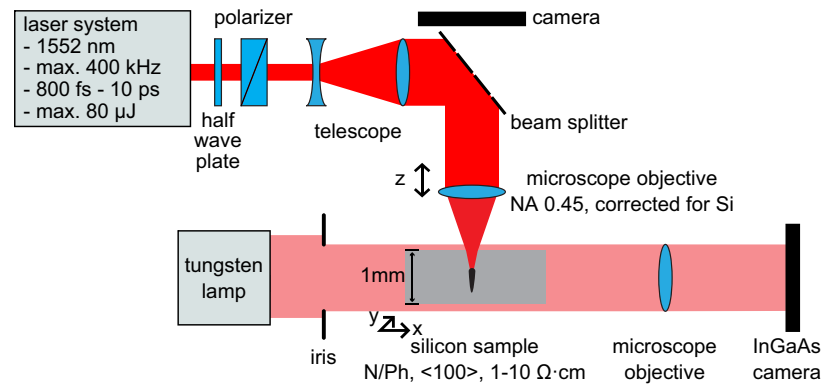

Fig. 1 Experimental setup for in-volume processing and in situ characterization

Table 1 Overview of processing parameters used for in-volume structuring of bulk silicon

\begin{tabular}{ll}
\hline Parameter & Investigated range \\
\hline Repetition rate & Single shot, $30-200 \mathrm{kHz}$ \\
Pulse duration & $0.8-10 \mathrm{ps}$ \\
Pulse energy on target & $0.7-6.7 \mu \mathrm{J}$ \\
Exposure time & $0.1-5 \mathrm{~s}$ \\
\hline
\end{tabular}

processing parameters is given in Table 1 . The pulse energy is varied by a combination of a half-wave plate and a polarizing cube. The laser beam is focused into the samples with the help of a microscope objective (Olympus LCPLN20XIR, $20 \times, \mathrm{NA}=0.45$ ) which is equipped with an adjustable collar for the correction of spherical aberrations inside silicon. In addition, a telescope is installed to adjust the laser beam diameter to the entrance aperture of the objective.

All experiments were performed inside low-doped silicon [n-type/Ph-doped, $\langle 100\rangle, 1-10 \Omega \cdot \mathrm{cm}]$ samples with a thickness of $1 \mathrm{~mm}$. To avoid damaging the surface, the focus position is chosen at a depth of $500 \mu \mathrm{m}$ below the front surface. In practice, to ensure constant and reproducible operating conditions, we did locate first the beam waist at the surface using combined $z$-scan and energy-scan techniques and plasma occurrence as feedback diagnostic (monitored by front surface imaging, see Fig. 1) to determine the $z$-coordinate of the surface. Afterwards the sample is moved with a precision stage to locate the beam waist at the center of the sample. The alignment of the processing region was realized with the help of a 3D positioning system (Aerotech, ANT 130) able of sub-micrometer accuracy.

The in situ interaction diagnostics is based on a customized infrared transmission microscope to observe the modifications in a direction perpendicular to the laser axis. A tungsten lamp is used for incoherent illumination. For imaging, a long distance NIR objective with a numerical aperture of 0.26 (10× magnification, Mitutoyo, Plan Apo) images the interior of the wafer onto an InGaAs camera (WiDy SWIR $320 \mathrm{U})$.

\section{Results and discussion}

\subsection{Below or near-threshold conditions in single-shot experiments}

At first, single-shot experiments were performed. We noticed that this processing regime does not lead to any modifications in Si for all tested pulse durations and energies (see Table 1). This might be due to limitations of the in situ observation or insufficient single-pulse intensities to achieve permanent modifications (maximum pulse energy $6.7 \mu \mathrm{J} /$ pulse on target). We estimate the spatial resolution of the in situ diagnostics to be $\sim 2.8 \mu \mathrm{m}$ considering the NA used for the imaging system and the central wavelength $(1.2 \mu \mathrm{m})$ of the illumination light transmitted through the Si sample. The achieved resolution is thus comparable to the focus diameter of the laser beam (estimated to be $\sim 4.8 \mu \mathrm{m}$ ) and to the expected transverse size of the eventual modifications inscribed by the laser. Therefore, our imaging setup should have been able to observe permanent local in-bulk modifications if the latter would occur. We presume that we do not reach the required energy density for macroscopic single-shot modifications. Indeed, losses during the propagation of the pulse before the focus, which are known as an important limitation in the ultrafast regime $[5,9]$, have to be considered limiting the energy deposition in the focal region.

\subsection{Exceeding the modification threshold in multi-pulse regime}

Further investigations are conducted in the multiple-shot regime to increase the accumulated illumination dose. We varied different laser parameters namely the repetition rate, pulse energy, number of pulses and pulse duration. Using this approach, permanent modifications localized in the bulk of silicon without damaging the front and back surfaces were detected for the first time. The rear and the front faces of the samples have been observed post-mortem with an optical microscope (ZEISS Axiotec) to confirm that there was no modification on the surfaces.

As an illustration, Fig. 2 shows a typical modification trace that was detected by our imaging diagnostics after 16,665 shots at $800 \mathrm{fs}$ (on part a) and at 10 ps (on part b). The processed regions are separated by a spacing of 100 $\mu \mathrm{m}$. The white markings in Fig. 2 indicate the different positions at the sample surface where the laser beam was coupled into the volume. Both cases show modifications confined inside the bulk. Although the geometrical focus is located at the middle of the sample and remains constant throughout the experiment, the starting point of the 


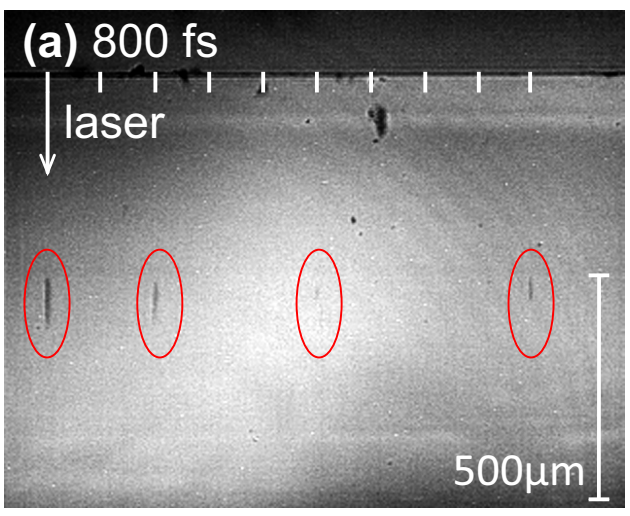

Fig. 2 Typical examples of the induced modifications in silicon using pulse durations of $800 \mathrm{fs}$ (a) and $10 \mathrm{ps}$ (b). Focus position is located at $500 \mu \mathrm{m}$ below the surface. The red dashed line schematically illustrates the beam caustic for an undisturbed beam. The other processing

modifications varies in its location along the laser beam axis by several tens of microns. Moreover, the modification grows towards the prefocal region and seems to saturate at a depth where the fluence is no more sufficient to modify the bulk of Si.

To our knowledge, this represents the first permanent modifications achieved inside silicon in the picosecond regime. While the shortest pulse duration of 800 fs does not lead to reproducible modifications as we will show later on, it is worth noting the very modest NA used for focusing $(\mathrm{NA}=0.45)$, which still leads to bulk modifications in this experiment in comparison to the severe focusing requirements $(\mathrm{NA}=3$ ) reported with the use of 100 -fs laser pulses [9].

\subsection{The absence of thermal accumulation on a pulse-to-pulse basis}

Recently, modifications have been induced inside Si with $250 \mathrm{fs}$ pulses at high repetition rate $(250 \mathrm{kHz})$ [11]. This encourages us to study the influence of the repetition rate and number of shots. To study the statistical behavior of the induced modifications we repeated the measurement with identical parameters several times at different sample sites in order to analyze the modification probability. Results are presented in Fig. 3 for 10 ps, and show the modification probability for two different conditions of irradiation: 100,000 shots at $200 \mathrm{kHz}$ and 16,665 pulses at $33 \mathrm{kHz}$. The difference between the two measurements is too low to demonstrate any influence of thermal accumulation between two pulses. The observed independence on the repetition rate supports the hypothesis that the material thermally fully relaxes between two shots even for the highest repetition rate investigated.

\section{(b) $10 \mathrm{ps}$}

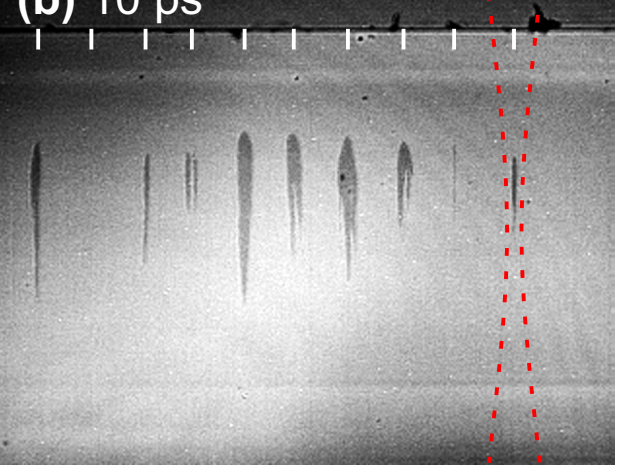

parameters are: $33.3 \mathrm{kHz}$ repetition rate, $6.6 \mu \mathrm{J}$ pulse energy and 0.5 $\mathrm{s}$ irradiation time (i.e., 16,665 pulses per spot). The red ellipses mark the induced modifications in the fs regime

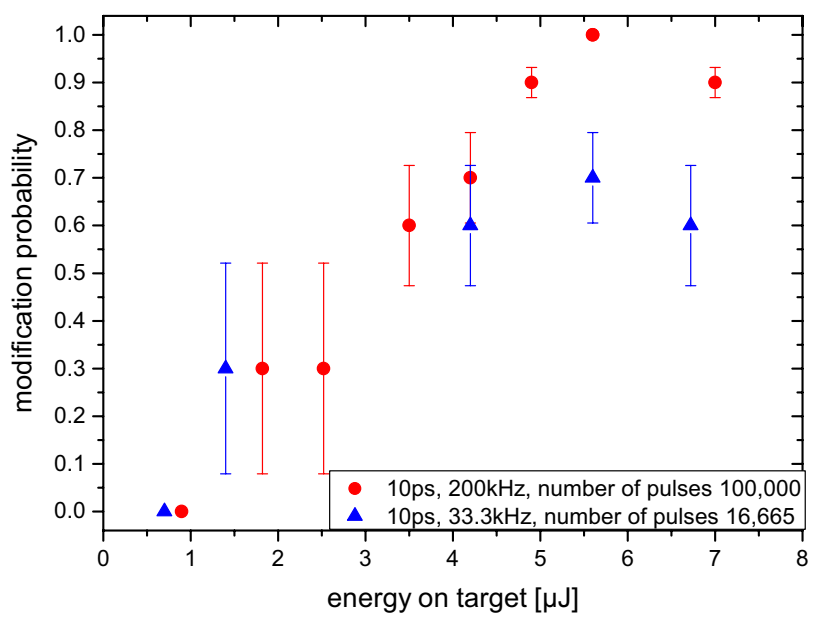

Fig. 3 Modification probability for different repetition rates and number of shots at $10 \mathrm{ps}$

Indeed, upon laser excitation and after the laser energy transfer from the electrons to the lattice taking place on a picosecond time scale $[12,13]$ the Si temperature rises in the excited volume before cooling through heat conduction. An estimation of the relaxation time is given by the heat diffusion time, $t_{\text {heat }}=l_{s}^{2} / D$, where $D$ is the thermal diffusivity of $\mathrm{Si}\left(\mathrm{in} \mathrm{cm}^{2} / \mathrm{s}\right.$ ) and $l_{s}$ accounts for the typical radial dimension on which the laser energy is deposited in the material. The thermal diffusivity of $\mathrm{Si}$ is temperature-dependent and tends to decrease with increasing temperature [14]. Even if we do not know the exact evolution of the temperature in the laserexcited volume, there is strong evidence that melting is not reached after one-shot irradiation. Considering the reduction of the thermal diffusivity when the lattice temperature approaches the melting temperature $\left(D=0.15 \mathrm{~cm}^{2} / \mathrm{s}\right.$ at 1414 $\mathrm{K}$ [14]) and a radial dimension of the laser-excited region 
equal to the beam radius $\sim 2.1 \mu \mathrm{m}$ ), we calculate a maximum cooling time being approximately $t_{\text {heat }} \sim 0.3 \mu \mathrm{s}$, which is an order of magnitude below the time separation between two shots considering the highest repetition rate $(200 \mathrm{kHz})$ used in our experiments. Therefore, accumulation of transient thermal effects due to insufficient relaxation between two shots is very unlikely in our operating conditions, but somehow in contradiction with the work of Pavlov et al. [11].

Nevertheless, regarding the statistical nature of our experiments we have to consider diverse accumulation processes which appear most likely in combination with nonlinear interaction. Within this discussion, the influence of pulseto-pulse instabilities of our laser system can be neglected due to pulse energy variations below $1 \%$. The absorption of the solid may be locally modified by the laser shot after shot. Besides, when a modification appears we assume that every shot modifies the structure of the material. So the consecutive pulses do not interact with a transiently excited material in phase of relaxation but more probably with a material fully relaxed but different from its initial state. It is thus probable, even if it can be so tiny that we cannot detect any modification with our diagnostic imaging setup, that material changes take place after single-shot irradiation.

\subsection{Dependence of modification initiation on the pulse duration}

In order to improve the understanding of the mechanisms initiating modifications, we investigated the dependency of the probability for the occurrence of modifications (for a constant irradiation dose) on the pulse duration. In Fig. 4, the bulk modification probability for a constant energy dose and number of shots at different pulse durations are reported. This experiment has been performed using a repetition rate

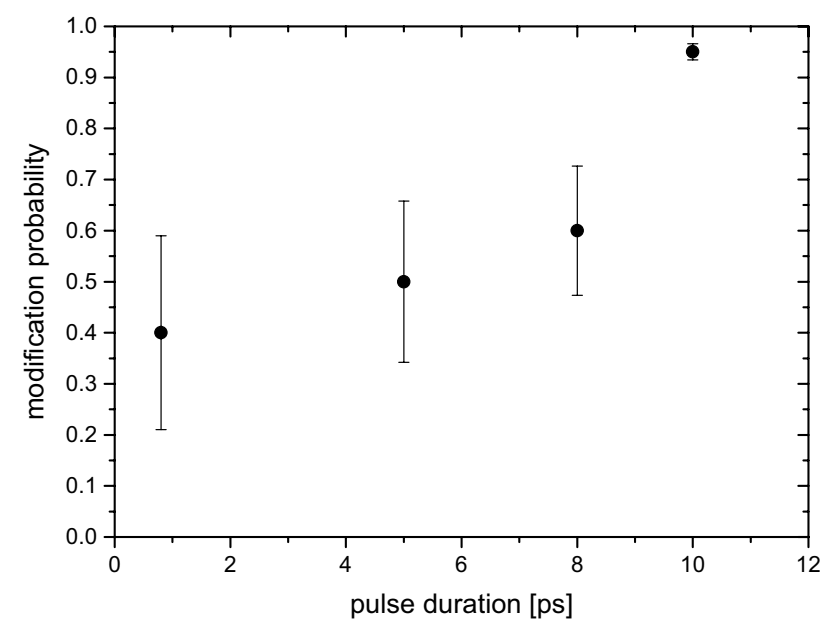

Fig. 4 Modification probability for $6.6 \mu \mathrm{J}$ at $30 \mathrm{kHz}(15,000$ shots $)$ for different pulse durations of $30 \mathrm{kHz}$ with 15,000 shots of $6.6 \mu \mathrm{J}$ each on target. Interestingly, the probability to induce an in-volume modification in silicon increases with pulse duration. This is counterintuitive, if considering the surface damage threshold which increases with pulse duration [15]. However, the main difference between bulk and surface modification mechanisms is the intensity clamping observed inside the bulk of $\mathrm{Si}[9$, 16]. Previous studies have shown that with the shortest pulse durations, the energy becomes very inefficiently delivered to the focus because of strong nonlinear propagation and plasma effects prior to the focus [9]. As a consequence, the required pulse energy to induce modifications is expected to be much higher than that for surface modification when very short pulses are applied. Accordingly, we assume that this very severe clamping mechanism in silicon leads to this unconventional pulse-duration dependence.

\subsection{Discussion}

The diversity in shape and size of the induced modifications with ps pulses implicates highly nonlinear interactions like self-focusing. Note, that the nonlinear refractive index for Si $n_{2} \approx 2.5 \times 10^{-18} \mathrm{~m}^{2} / \mathrm{W}$ is two orders of magnitude higher compared to typical values for materials like fused silica or other dielectrics [17, 18]. Accordingly, the critical power for self-focusing in silicon at a wavelength around $1550 \mathrm{~nm}$ is approximately $P_{\text {crit }} \approx 4 \times 10^{4} \mathrm{~W}$. Referring to the applied pulse energies within our experiments, the launched peak power into silicon was ten to hundred times higher $\left(P_{\text {peak }}>\right.$ $10^{5}-10^{6} \mathrm{~W}$ ) than this critical threshold. Moreover, filamentation was also observed, which is a direct evidence of highly disturbed intensity distributions within the focal region. The formation of distinct branches can be clearly seen in Fig. $2 b$ which could be the result of highly disturbed propagation within the focal region.

For pulse durations in the fs regime nonlinear effects are more pronounced than in the ps regime. They can lead to strong prefocal losses and perturbation of the propagation, resulting in high energy losses. Due to the high losses because of the nonlinear interaction, the modification threshold is exceeded only in a small region, creating confined modifications (see Fig. 2a). Since the structures are written with parameters close to the modification threshold, local material properties, defects, dust particles, etc. have a greater impact on the writing process.

When the pulse duration is increased, the sensitivity to nonlinear propagation effects (multi-photon ionization, Kerr effect) slightly diminishes as the peak power is getting smaller. Moreover, the influence of avalanche ionization compared to multi-photon absorption significantly increases for longer pulse lengths during the transition from fs to $\mathrm{ps}$ pulses as discussed in [19]. In addition, thermal effects may play an increased role as more energy can be transferred to 
the lattice during the pulse duration. This increase in lattice temperature in turn might change the absorption properties significantly due to bandgap closure.

As the bandgap closure depends on the lattice temperature, it is important to consider whether the lattice temperature is significantly increased during the pulse duration. While the pulse energy initially leads to the creation of an electron-hole plasma in the bulk material, the energy transfer to the lattice is driven by electron-phonon collisions. The characteristic time for equilibration can be estimated by [12]: $t_{e-p h}=\frac{\hbar M}{J_{i} m_{e}}$, with $J_{i}$ the ionization potential and $M$ and $m_{e}$ the atomic silicon and electron mass, respectively. For silicon $\left(J_{i}=8.15 \mathrm{eV}, M=28.0855 \mathrm{u}\right)$, the calculation yields about $4 \mathrm{ps}$, which means that temperature equilibration between electrons and lattice and further subsequent increase of lattice temperature should be observed before the pulse termination for pulses with a few ps duration. Note that a twotemperature model would provide better accuracy for estimating the temperature equilibration time between electrons and lattice, as well as the temperature evolution and associated diffusion, recombination, and thermal properties of silicon. However, this type of modeling of the relaxation and transport of the deposited energy of the electron subsystem requires a precise measurement of the energy deposition, which was beyond the scope of our experiments. Nevertheless, we can safely assume that the magnitude of the relaxation time is picoseconds, suggesting that lattice heating plays a role in the interaction [20].

The local heating can be estimated with very simple thermodynamic considerations: $\Delta T\left({ }^{\circ} \mathrm{C}\right)=E /(V \rho C)$, where $E$ is the pump energy, $V$ the interaction volume, $\rho$ the Si density and $C$ the specific heat capacity. For this calculation, an average cylinder volume was estimated by the length and width of the modifications seen in Fig. 2 for 10 ps. Note that this volume largely exceeds the confocal volume related to the laser, but it is representative of the volume where significant absorption takes place. Estimating a cylinder with a length of $300 \mu \mathrm{m}$ and $10 \mu \mathrm{m}$ of radius, with $\rho=2.33 \mathrm{~g} / \mathrm{cm}^{3}$, $\mathrm{C}=0.712 \mathrm{~J} / \mathrm{g}{ }^{\circ} \mathrm{C}$ and full absorption of the incoming energy, we find $\Delta \mathrm{T}=550 \mathrm{~K}$. This temperature increase leads to significant bandgap changes due to different atomic spacings. The temperature-dependent bandgap can be calculated using $E_{g}(T)=E_{g}(0)-\alpha T^{2} /(T+\beta)$, where $E_{g}(0)=1.166 \mathrm{eV}, \alpha=$ $4.7310^{-4} \mathrm{eV} / \mathrm{K}$ and $\beta=636 \mathrm{~K}$ are fit parameters [21].

Accordingly, a temperature increase of $550 \mathrm{~K}$ would lead to a reduced bandgap of $0.93 \mathrm{eV}$, while a reduction to the photon energy of $0.8 \mathrm{eV}$ (equivalent to the wavelength of $1.55 \mu \mathrm{m}$ ), where one would expect linear absorption to occur, would require a temperature increase of $\sim 900 \mathrm{~K}$. Nevertheless, these simple estimations of the local heating induced by ps laser pulses underline the role of thermal effects contrary to the femtosecond regime [5] and are consistent with a numerical study of laser-induced modifications in semiconductors where the importance of the lattice heating for picosecond pulses is shown [22].

In summary, avalanche ionization and bandgap closure are possible explanations for the observed modifications in the ps range. Furthermore, nonlinear effects that have less influence than in the fs range cannot be completely excluded.

\section{Conclusion}

Within this work, we successfully demonstrated in-volume structuring of silicon using ultrashort laser pulses in the range from 0.8 to $10 \mathrm{ps}$. A qualitative analysis of the induced modifications depending on the processing parameters repetition rate, pulse duration, pulse energy and pulse number was presented. The structures observed are a result of the cumulative action of many pulses. However, heat accumulation effects can be canceled out for the investigated pulse-to-pulse separations of at least $5 \mu$ s due to the high diffusivity of silicon. The expected growing influence of avalanche ionization and bandgap closure with increasing pulse duration may play an important role in these observations. Moreover, the high value of the nonlinear refractive index $n_{2}$ suggests a highly distorted intensity distribution at the focal region, even in the picosecond regime investigated here. This represents another factor that can potentially decrease the controllability of the interactions. In particular, this may explain the observation of multiple branches within the induced structures, but it requires more investigations to conclude on this aspect.

Moreover, the laser-written structures exhibit highly elongated modifications along the inscribing laser beam axis as well. In order to fabricate buried optical elements for future applications, pulse durations of a few hundred femtoseconds seem to be most suitable. However, the elongated characteristic and the statistical fluctuations in size prevent inscribing techniques perpendicular to the incident laser beam axis at the current state. These results support the recent work from Pavlov et al., where the fabrication of buried optical waveguides along the inscribing laser axis was demonstrated [11]. In order to improve the involume processing in general, the governing processes resulting from nonlinear interaction should be further investigated. Here, pump-probe experiments should offer a deeper insight into the spatial-temporal behavior and might lead to further optimized laser parameters or focusing strategies. Single-shot modification investigations are essential to improve our understanding of the physical processes involved in the initiation of the bulk modifications reported in this work. 
Acknowledgements This project has received funding from the European Research Council (ERC) under the European Union's Horizon 2020 research and innovation program (Grant Agreement No. 724480).

Open Access This article is distributed under the terms of the Creative Commons Attribution 4.0 International License (http://creativeco mmons.org/licenses/by/4.0/), which permits unrestricted use, distribution, and reproduction in any medium, provided you give appropriate credit to the original author(s) and the source, provide a link to the Creative Commons license, and indicate if changes were made.

\section{References}

1. K.M. Davis, K. Miura, N. Sugimoto, K. Hirao, Writing waveguides in glass with a femtosecond laser. Opt. Lett. 21(21), 1729 (1996)

2. R.R. Gattass, E. Mazur, Femtosecond laser micromachining in transparent materials. Nat. Photonics 2(4), 219-225 (2008)

3. V.V. Kononenko, V.V. Konov, E.M. Dianov, Delocalization of femtosecond radiation in silicon. Opt. Lett. 37(16), 3369 (2012)

4. A. Mouskeftaras, A.V. Rode, R. Clady, M. Sentis, O. Utéza, D. Grojo, Self-limited underdense microplasmas in bulk silicon induced by ultrashort laser pulses. Appl. Phys. Lett. 105, 191103 (2014)

5. E.V. Zavedeev, V.V. Kononenko, V.I. Konov, Delocalization of femtosecond laser radiation in crystalline $\mathrm{Si}$ in the mid-IR range. Laser Phys. 26, 016101 (2016)

6. A.H. Nejadmalayeri, P.R. Herman, J. Burghoff, M. Will, S. Nolte, A. Tünnermann, Inscription of optical waveguides in crystalline silicon by mid-infrared femtosecond laser pulses. Opt. Lett. 30(9), 964 (2005)

7. Y. Ito, H. Sakashita, R. Suzuki, M. Uewada, K.P. Luong, R. Tanabe, Modification and machining on back surface of a silicon substrate by femtosecond laser pulses at $1552 \mathrm{~nm}$. J. Laser Micro Nanoeng. 9(2), 98-102 (2014)

8. M. Chambonneau, Q. Li, M. Chanal, N. Sanner, D. Grojo, Writing waveguides inside monolithic crystalline silicon with nanosecond laser pulses. Opt. Lett. 41(21), 4875 (2016)

9. M. Chanal, V.Y. Fedorov, M. Chambonneau, R. Clady, S. Tzortzakis, D. Grojo, Crossing the threshold of ultrafast laser writing in bulk silicon. Nat. Commun. 8, 773 (2017)
10. O. Tokel, A. Turnal, G. Makey, P. Elahi, T. Olakolu, E. Ergeen, O. Yavuz, R. Hübner, M.Z. Borra, I. Pavlov, A. Bek, R. Turan, D.K. Kesim, S. Tozburun, S. Ilday, F. Ö. Ilday, In-chip microstructures and photonic devices fabricated by nonlinear laser lithography deep inside silicon. Nat. Photonics 11, 639645 (2017)

11. I. Pavlov, O. Tokel, S. Pavlova, V. Kadan, G. Makey, A. Turnali, Ö. Yavuz, F.Ö. Ilday, Femtosecond laser written waveguides deep inside silicon. Opt. Lett. 42(15), 3028-3031 (2017)

12. B. Luther-Davies, A.V. Rode, N.R. Madsen, E.G. Gamaly, Picosecond high-repetition-rate pulsed laser ablation of dielectrics: the effect of energy accumulation between pulses. Opt. Eng. 44(5), $051102(2005)$

13. H.M. Van Driel, Kinetics of high-density plasmas generated in Si by $1.06-$ and $0.53-p m$ picosecond laser pulses. Phys. Rev. B 35(15), 8166 (1987)

14. H.R. Shanks, P.D. Maycock, P.H. Sidles, G.C. Danielson, Thermal conductivity of silicon from 300 to $1400 \mathrm{~K}$. Phys. Rev. B 130(5), $1743(1963)$

15. X. Wang, Z.H. Shen, J. Lu, X.W. Ni, Laser-induced damage threshold of silicon in millisecond, nanosecond, picosecond regimes. J. Appl. Phys. 108, 033103 (2010)

16. D. Grojo, A. Mouskeftaras, P. Delaporte, S. Lei, Limitations to laser machining of silicon using femtosecond micro-Bessel beams in the infrared. J. Appl. Phys. 117, 153105 (2015)

17. A. Couairon, A. Mysyrowicz, Femtosecond filamentation in transparent media. Phys. Rep. 441, 47-189 (2007)

18. Q. Lin, J. Zhang, G. Piredda, R.W. Boyd, P.M. Fauchet, G.P. Agrawal, Dispersion of silicon nonlinearities in the near infrared region. Appl. Phys. Lett. 91, 021111 (2007)

19. B. Rethfeld, Free-electron generation in laser-irradiated dielectrics. Phys. Rev. B 73, 035101 (2006)

20. B. Rethfeld, D.S. Ivanov, M.E. Garcia, S.I. Anisimov, Modelling ultrafast laser ablation. J. Appl. Phys. 50, 193001 (2017)

21. V. Alex, S. Finkbeiner, J. Weber, Temperature dependence of the indirect energy gap in crystalline silicon. J. Appl. Phys. 79, 6943 (1996)

22. J.K. Chen, D.Y. Tzou, J.E. Beraun, Numerical investigation of ultrashort laser damage in semiconductors. Int. J. Heat Transf. 48, 501-509 (2015) 